\title{
Frequency of Methicillin-resistant Staphylococcus aureus Nasal Colonization among preschool and school children under 14 years old in Urmia
}

\author{
Ebrahim Sadeghi', Amir Nasim Far', Mohammad Karamiyat', Ahad Gazzavi', Ahmad Ali Nikibakhsh', Mehran Noroozi ${ }^{\dagger}$
}

Received 22 Dec 2016, Accepted for publication 16 Feb, 2017

\begin{abstract}
Background and Aims: Nasal Colonization with Methicillin Resistant Staphylococcus aureus (MRSA) is known as an important risk factor in the development of dangerous infections.

Materials and Methods: The present study seeks to investigate the prevalence of MRSA nasal colonization among the preschool and school children under 14 in Urmia. To do so, specimens for culture were obtained from 400 children. Cultures and antibiograms with oxacillin discs were performed in Shahid Motahari hospital of Urmia.

Results: 81 cases (47 females, 34 males) out of 400, nasal colonization were observed. Of which 12 (5 females, 7 males) were resistant to methicillin. Colonization was slightly higher among the females with no statistically significant difference (P.value>1).

Conclusion: The results indicate that colonization with MRSA is present among healthy children in the studied population. Fortunately, the respective prevalence was not higher compared to other areas. Further attention is required by the health authorities to prevent the transmission of such organisms among children.
\end{abstract}

Key Words: Staphylococcus aurous, Methicillin Resistant, nasal colonization

Address: Urmia, Urmia University of Medical Sciences, Shahid Motahari hospital

Tel: (+98) 9143492885

Email: sadeghi.e@umsu.ac.ir

\section{Introduction}

Staphylococcus Aureus is among the first known pathogenic organisms in humans which causes life threatening, invasive and local infections in all age groups. The toxin produced by this bacterium can result toxic shock syndrome and food poisoning (1). Studies have shown that Staphylococus aureus (S.aureus) carriers pose an important risk factor for the development of secondary bacterial infections
(2). The organism can colonize in the skin and in the nose temporarily and transmitted between individuals directly or indirectly through contaminated people, objects and asymptomatic carriers (3). According to the reports, more than $\% 80$ of the organisms causing Staph infections originate from the nose and its elimination from this site can help reduce the colonization in other sites of the body $(4,5)$.

\footnotetext{
${ }^{1}$ Assistant Professor of Pediatric Infectious Diseases, Urmia University of Medical Sciences (Corresponding Author)

${ }^{2}$ Assistant Professor of Pediatric Infectious Diseases, Urmia University of Medical Sciences

${ }^{3}$ Associate Professor of Pediatric Infectious Diseases, Urmia University of Medical Sciences

${ }^{4}$ Assistant Professor of Neurology, Urmia University of Medical Sciences

${ }^{5}$ Professor of Pediatrics, Urmia University of Medical Sciences

${ }^{6}$ Assistant Professor of children's blood, Urmia University of Medical Sciences
} 
This organism became a serious health concern, especially among hospitalized patients when it was found to be resistant to all beta-lactamase drugs by the 1960s. Since the late 1990s, there have been reports of community acquired infections with $\mathrm{S}$ aureus strains in individuals with no risk factors and hospitalization history. Nevertheless, the prevalence rate is on the rise in different parts of the world, with more frequent MRSA (Meticillin Resistant S.aureus) isolates from carriers with history of hospital stay and risk factors (6).

Accordingly, the epidemiology of colonization with MRSA strains has changed and is no longer limited to hospitalized patients or people with underlying risk factors. Since the identification of MRSA, many studies were conducted throughout the world to determine the prevalence and risk factors associated with the organisms, all indicating significant differences in the prevalence among various countries and even increasing rate in countries with a low prevalence rate (7).

Given that nasal colonization with MRSA poses a risk factor of infection in the carriers and its transmission to other community members, and lack of sufficient data about its prevalence in Urmia, the present study seeks to determine the prevalence of MRSA nasal colonization in healthy children and respective antibiotic resistance

\section{Materials and Methods}

In total, 400 swab samples from randomly selected preschool and school children (female and male) were collected and entered into the study. Demographic data, including age, gender, family details (i.e., number of family members, parents' occupations, history of chronic diseases in the family) and risk factors were collected using a questionnaire. Exclusion criteria were having parents working in health centers, history of hospitalization during the past six months, antibiotic use during the last month, and history of chronic diseases such as asthma, diabetes, cystic fibrosis and kidney diseases.

Swab samples were collected from the anterior nasal cavity by the researchers using cotton swabs soaked in normal saline and rotated five times in the nostrils. They were then stored in Stuart transport medium (Merk, Germany). The samples were transferred to the microbiology division of Shahid Motahary hospital and cultured on Mannitol Salt Agar (TIAN BIOTECH, India).

This medium was used since it contains Sodium Chloride \%7.5 and has a growth inhibitory effect on other organisms so that it can be used for respiratory clinical samples. Since Mannitol is present in the medium which can be fermented specifically by Saureus, we used it for both culture and microbial identification.

The samples were incubated for $24-48$ hours at $35^{\circ} \mathrm{C}$ and then studied. The grown yellow color colonies, indicator of Manittol fermentation, were selected for catalase coagulase test (8). The confirmed colonies were isolated for meticillin sensitivity test. In doing so, we used Mueller-Hinton Agar medium (Tian Biotech, India) and Oxacillin $1 \mathrm{mcg}$ disk (Padtan teb, Iran).

\section{MRSA Examination:}

Bacterial suspension of colonies adjusted to \%0. 5 McFarland were selected from an 18-24 hour. Then, a sterile swab was dipped into the suspension and re-cultured on the Mueller Hinton agar plate after drying excess water. After 15 minutes, oxacillin $1 \mathrm{mcg}$ disk was placed on the plate and incubated at $35^{\circ} \mathrm{C}$ for $18-24$ hours. Zone diameter was measured using a millimeter ruler and results were interpreted according to CLSI guideline. Inhibition zone of $<10$ was considered as oxacillin 
resistant strains. (Due to the unavailability of meticillin disk, we used oxacillin disk) (8).

\section{Results}

In total, 400 samples consisting of 200 female and 200 male children under the age of 14 years were used in the study. Of the 200 male samples, 34 (\%17) were detected with $\mathrm{S}$.aureus; 2 in 1-6 years age group, 11 in 7-10 years group, and 15 in 11-14 years group. Of 200 female samples, $\mathrm{S}$ aureus were observed in 47 (\%23.5); 8 in 1-6 years age group, 17 in 7-10 years group, and 22 in 11-14 years age group. Among 34 positive $\mathrm{S}$ aureus male samples, 7 ( \%20.6) were found to be meticillin resistant type, of which 2 were in 1-6 years age group, 2 in 7-10 years age group, and 3 in 11-14 years age group. Of the 47 positive female samples, $5(\% 10.6)$ were meticillin resistant S.aureus, consisting of 1 in 1-6 years age group, 2 in 7-10 years age group, and 2 in 11-14 years age group. No statistically significant difference was observed in age and sex distributions among the positive culture results $(\mathrm{P}=0.1)$.

Table 1. Age distribution in Metacillin resistant $\mathrm{S}$ aureus carriers

\begin{tabular}{lllll} 
negative & \multicolumn{5}{l}{ positive } & gender \\
\hline Methicillin resistant type & & & Age group & \\
\hline Frequency(\%) & Age group & Frequency(\%) & $1-6$ & male \\
\hline $6(75)$ & $1-6$ & $2(25)$ & $7-10$ & \\
$9(81.8)$ & $7-10$ & $2(18.2)$ & $11-14$ & female \\
$12(80)$ & $11-14$ & $3(20)$ & $1-6$ & \\
\hline $7(87.5)$ & $1-6$ & $1(12.5)$ & $7-10$ & \\
$15(88.2)$ & $7-10$ & $2(11.8)$ & $11-14$ & \\
$20(90.9)$ & $11-14$ & $2(9.1)$ & & \\
\hline
\end{tabular}

Table 2. Frequency of resistant strains in culture samples based on sex distribution

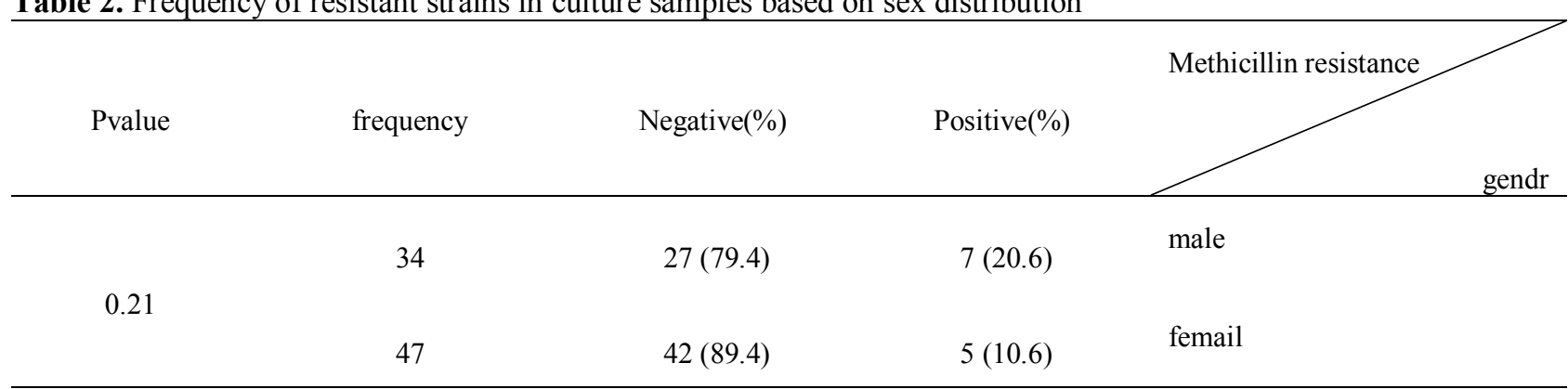

\section{Discussion}

$\mathrm{S}$ aureus is among the most notorious infective agents in the community and hospital environments. In recent decades, the corresponding infection has increased. After the emergence of resistance to
Penicillin, beta lactamase penicillins such as meticillin were introduced for the first line treatment of the infections caused by $\mathrm{S}$ aureus. The first reported case of MRSA was a year after metacillin administration 9. Since then, the 
prevalence of MRSA augmented steadily till it was reported in 1990 as widespread throughout the world (9-10).

MRSA strains were thought for long to be a causative agent of hospital acquired infections; however, recent reports from different parts of the world suggest that the organism can strike healthy people as well. Nostrils in children and adults act as the main locus for the organism so that carriers play a critical role in both hospital and community acquired infections $(2,4)$.

A comprehensive analysis has been done on the existing reports about MRSA in the world. In this study, CA-MRSA strains were assigned to two groups; one with no risk factor and the other with the risk factor. The frequency was low in the former, while it was higher in the latter, i.e, hospitalized patients or those with the risk factor. The difference in the world reports could be explained by the lack of a clear standard cut definition for CA-MRSA. Besides, some studies are indicative of rising trends in the prevalence of the organism in recent years in some parts of the world (17-19).

In a study by Creech et al, in Nashville, USA, a 10 fold increase was evident in the prevalence of CAMRSA colonization among children over a 3 year period (\%0.8 in 2002 vs, \%9.2 in 2004). PCR assays were performed in the present study and 7 cases of MRSA strains were revealed to have mecA gene, however, in 4 cases the gene was absent despite being meticillin resistant, which could be explained by different diagnostic methods used for microbial identification. It is worth mentioning that PCR has been considered as the gold standard in microbial characterization in different studies $(20$, 21).

In a study by Bingardi et al, $\% 50$ of $\mathrm{S}$ aureus were found meticillin resistant by disk method, but PCR assay revealed $\% 48.2$, indicating false positive results produced by disk method for the strains containing heterogeneous resistance (20). On the other hand, some reports show intermediate or low resistance to Meticillin but sensitivity to Co amoxiclav in the strains without mecA (21).

One of the problems is the lack of a clear definition for Borderline Resistant Staphylococcus Aureus, and consequently a range of prevalence were reported $(\% 0.9-\% 12.5)$. It seems the reason for resistance in such cases is a beta lactamase production (21).

The results of the present study are suggestive of the presence of colonization with MRSA in the study region among healthy children with no serious risk factors. Fortunately, the prevalence of CA-MRSA is not high, compared to that in other areas. In conclusion, more attention is needed by health authorities and health care providers to prevent the transmission of resistant strains.

Important contributing factors for MRSA transmission include overcrowding, frequent skin contact, lack of hygiene, and use of contaminated stuffs. Given that $\mathrm{S}$ aureus infections are life threatening, continuous monitoring and surveillance of antibiotic resistance, education for health staff and tracking patients are warranted. Standard care for the hospitalized patients and hand washing, especially in intensive care units should be pursued steadily. Prudent antibiotic prescription for MRSA is very helpful and further gene based studies on resistance are highly recommended.

\section{References}

1. Huang Y-C, Hwang K-P, Chen P-Y, Chen C-J, Lin TY. Prevalence of methicillin-resistant Staphylococcus aureus nasal colonization among Taiwanese children in 2005 and 2006. J Clin Microbiol 2007;45(12): 3992-5.

2. White A. Increased infection rates in heavy nasal carries of coagulase positive staphylococci.Anti 
microbial agentschemother. Pediatr Infect Dis J 1963; 161: 667-80.

3. Wertheim HF, Vos MC, Ott A, van Belkum A, Voss A, Kluytmans JA et al. Risk and outcome of nosocomial Staphylococcus aureusbactermia in nasal carries versus non carries. Lancet 2004;364: 703-5.

4. Reagan DR, Doebbeling BN, Pfaller MA, Sheetz CT, Houston AK, Hollis RJ, et al. Elimination of coincident Staphylococcus aureus nasal and hand carriage with intra nasal application of mupirocin calcium ointment. Ann intern med 1991;114: 101-6.

5. Salmenlinna S, Lyytikäinen O, Vuopio-Varkila J. Community acquired methicillin resistant Staphylococcus aureus Finland. Emerg infect Dis 2002;8(6): 602-7.

6. Nakamura MM, Rohling KL, Shashaty M, Lu H, Tang YW, Edwards KM. Prevalence of methicillin resistant Staphylococcus aureus nasal carriage in the community pediatric population. Pediatr infec Dis J 2002;21: 917-21.

7. Hussain FM, Boyle-Vavra S, Daum RS. Community acquired methicillin resistant Staphylococcus aureus colonization in healthy children attending an outpatient pediatric children. Pediatr infec Dis J 2001;20: 763-7.

8. Felten A, Grandry B, Lagrange PH, Casin I. Evaluation of three method for detection of low level Methicilline Resistant Staphylococcus aureus: a disk diffusion method cefotaxime and moxalactam. J Clin Micbiol 2002;40: 2766-71.

9. Rijal KR, Pahari N, Shrestha BK, Nepal AK, Paudel B, Mahato $\mathrm{P}$, et al. Prevalence of methicillin resistant Staphylococcus aureus in school children of pokhara. Nepal med coll J 2008;10(3): 192-5.

10. -Heininger U, Datta F, Gervaix A, Schaad UB, Berger C, Vaudaux B,etal.Prevalance of methicillin resistant Staphylococcus aureus nasal colonization in children a multicenter cross sectional study. Pediatr infect Dis J 2007;26(6): 546-4.

11. Sedighi I, Moez HJ, Alikhani MY. Nasal carriage of methicillin resistant Staphylococcus aureus and their antibiotic susceptibility patterns in children attending day-care centers. Acta Microbiol Immunol Hung 2011;58(3):227-34.

12. Sharifi M, Karimzadeh T, Mohammadi-Chelkasari F, Bijani B, Alipoor-Heydari M. Community-acquired methicillin-resistant Staphylococcus aureus: prevalence and risk factors. JQUMS 2009;12:75-82.

13. Creech CB, Kernodle DS, Alsentzer A, Wilson C, Edwards KM. Increasing rates of nasal carriage of methicillin resistant Staphylococcus aureus in healthy children. Pediatr infect Dis J 2005;24(7): 617-622

14. Fritz SA, Garbutt J, Elward A, Shannon W, Storch GA. Prevalence of and risk factor for community pha acquired methicillin resistant and methicillin sensitive staphylococcus aureus colonization in children seen in a practice based research network. Pediatr 2008; 121: 6-8.

15. Suggs AH, Maranan MC, Boyle-Vavra S, Daum RS.Methicilline Resistant and Borderline Resistanta symptomatic Staphylococcus aureus Colonization in children without identifical risk factors. PediatrInf Dis J 1999;18: 410-4.

16. Masuda K, Masuda R, Nishi J-I, Tokuda K, Yoshinaga M, Miyata $\mathrm{K}$. Incidences of nasopharyngeal colonization of respiratory bacterial pathogens in Japanese children attending day-care centers. Pediatr Int 2002;44(4):376-80.

17. Oguzkaya-Artan M, Baykan Z, Artan C. Nasal carriage of Staphylococcus aureus in healthy Preschool Childrens. Jpn J Inf Dis 2008;61: 70-2.

18. Kallen AJ, Driscoll TJ, Thornton S, Olson PE, Wallace MR. Increase in Community acquired methicillin resistant Staphylococcus aureus at a naval medical center. Inf Cont Hosp Epidemiol 2000;21: 223-6. 
19. Manju MP, Ragunathan L, Gautam S. Detection of Methicillin Resistance in Staphylococcus Aureus by Polymerase Chain Reaction and Conventional Methods: A Comparative Study. J Lab Physicians 2012; 4(2): 83-8.

20. Bignardi GE, Woodford N, Chapman A, Johnson AP, Speller DC. Detection of mecA gene and phenotypic Detection of resistant in Staphylococcus aureus isolates borderline or low level MethicillineResistant. J Antimicrob Chemother 1996;37: 53-63.

21. Hussain FM, Boyle-Vavra S, Daum RS. Community acquired methicillin resistant Staphylococcus aureus Colonization in healthy Childrens an outpatient pediatric clinic. Pediatr Inf Dis 2002;20: 763-7. 